\title{
A-MnasNet: Augmented MnasNet for Computer Vision
}

\author{
Prasham Shah \\ IoT Collaboratory at IUPUI \\ Department of Electrical and Computer Engineering \\ Purdue School of Engineering and Technology \\ Indianapolis, USA \\ pashah@purdue.edu
}

\author{
Mohamed El-Sharkawy \\ IoT Collaboratory at IUPUI \\ Department of Electrical and Computer Engineering \\ Purdue School of Engineering and Technology \\ Indianapolis, USA \\ melshark@iupui.edu
}

\begin{abstract}
Convolutional Neural Networks (CNNs) play an essential role in Deep Learning. They are extensively used in Computer Vision. They are complicated but very effective in extracting features from an image or a video stream. After AlexNet [5] won the ILSVRC [8] in 2012, there was a drastic increase in research related with CNNs. Many state-of-theart architectures like VGG Net [12], GoogleNet [13], ResNet [18], Inception-v4 [14], Inception-Resnet-v2 [14], ShuffleNet [23], Xception [24], MobileNet [6], MobileNetV2 [7], SqueezeNet [16], SqueezeNext [17] and many more were introduced. The trend behind the research depicts an increase in the number of layers of CNN to make them more efficient but with that the size of the model increased as well. This problem was fixed with the advent of new algorithms which resulted in a decrease in model size. As a result, today we have CNN models which are implemented on mobile devices. These mobile models are small and fast which in turn reduce the computational cost of the embedded system. This paper resembles similar idea, it proposes a new model Augmented MnasNet (A-MnasNet) which has been derived from MnasNet [1]. The model is trained with CIFAR-10 [4] dataset and has a validation accuracy of $96.89 \%$ and a model size of $11.6 \mathrm{MB}$. It outperforms its baseline architecture MnasNet which has a validation accuracy of $80.8 \%$ and a model size of $12.7 \mathrm{MB}$ when trained with CIFAR-10.
\end{abstract}

Index Terms-Convolutional Neural Networks (CNNs), Computer Vision, A-MnasNet, CIFAR-10, MnasNet.

\section{INTRODUCTION}

Convolutional Neural Networks have become an integral part of Computer Vision. They are extensively used for Computer Vision applications like Image Classification [19], Object Detection [21] and Semantic Segmentation [20]. As these models become deeper, they need more computation and as a result they become slower. This makes it difficult to deploy them on resource-constrained platforms.

Mobile devices have limited resources so the prime focus of researchers has been on designing compact models with a fair trade-off between accuracy and computational cost. A new class of CNN model was proposed in May 2019 by Google Brain and Google Inc known as MnasNet. They introduced a neural architecture search approach which optimized accuracy and latency on mobile devices. It outperforms other novel architectures like MobileNetV2, NASNet [30] and ResNet-50 $[12]$ in terms of speed and accuracy.

\section{PRIOR WORK}

The research has been mainly focused on improving resource efficiency of CNN models. Weight initialization, learning rates, activation functions, network topology, batches and epochs, regularization and optimization are different parameters that affect the efficiency of model. In this paper, first baseline architecture and then the new architecture with pro-

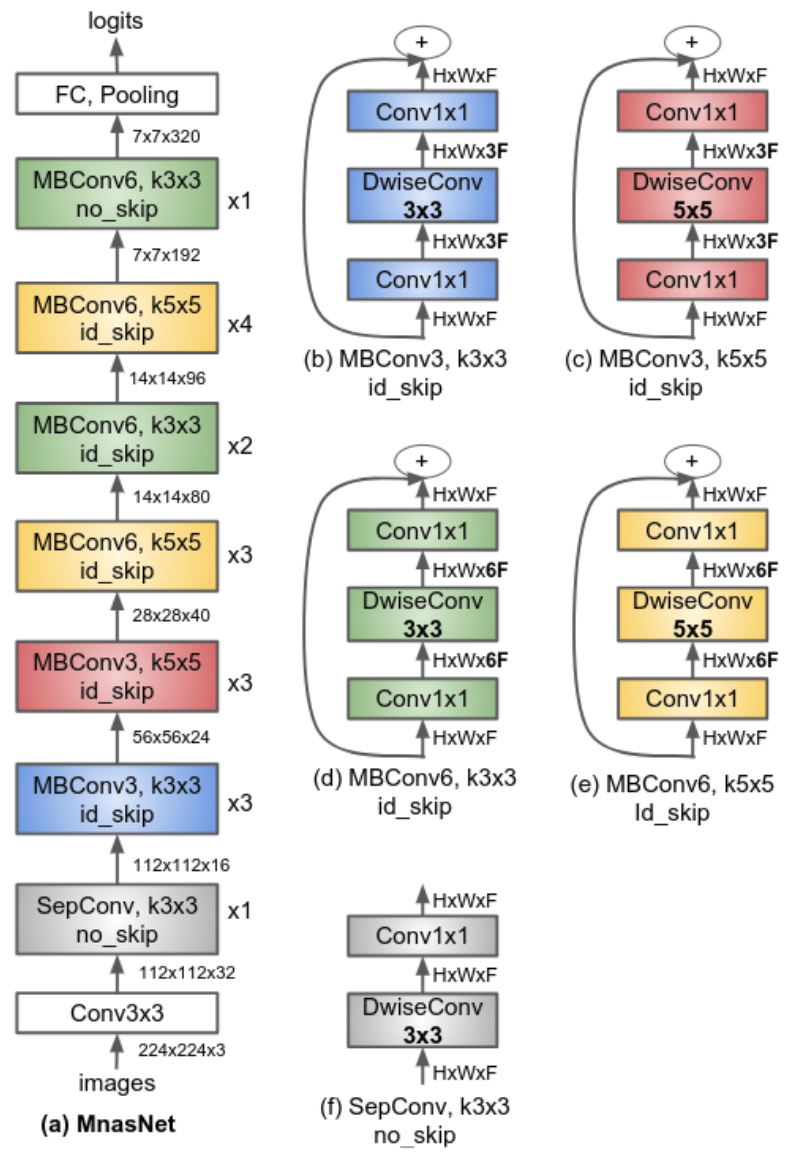

Fig. 1. MnasNet architecture

This is the author's manuscript of the article published in final edited form as: 
posed changes have been discussed.

\section{A. MnasNet Architecture}

In this paper, they have introduced a neural architecture search approach which optimized accuracy and latency on mobile devices using reinforcement learning. By using their automated approach, they propose various architectures called MnasNet-A1, MnasNet-A2 and MnasNet-A3. They show that diversity of layers in such resource-constrained models yield better trade-offs between accuracy and latency of the model. They have shown that their architecture outperforms other models like MobileNetV1, SqueezeNext, ShuffleNet, MobileNetV2, NASNet and many other models.

As shown in Figure 1, MnasNet uses Convolution Ops, depthwise separable convolution, mobile inverted bottleneck layers to extract features. It uses RMSProp optimizer [31], Batch Normalization [27] and Dropout regularization [32]. Table I shows the MnasNet architecture which is trained with CIFAR-10 dataset.

\begin{tabular}{|c|c|c|c|c|c|}
\hline \multicolumn{6}{|c|}{ MnasNet Architecture } \\
\hline Layers & Convolutions & $\mathrm{t}$ & $\mathrm{c}$ & $\mathrm{n}$ & $\mathrm{S}$ \\
\hline $32^{2} \times 3$ & Conv2d $3 \times 3$ & - & 32 & 1 & 1 \\
\hline $112^{2} \times 32$ & SepConv $3 \times 3$ & 1 & 16 & 1 & 2 \\
\hline $112^{2} \times 16$ & MBConv3 $3 \times 3$ & 3 & 24 & 3 & 2 \\
\hline $56^{2} \times 24$ & MBConv3 $5 \times 5$ & 3 & 40 & 3 & 2 \\
\hline $28^{2} \times 40$ & MBConv6 $5 \times 5$ & 6 & 80 & 3 & 2 \\
\hline $14^{2} \times 80$ & MBConv6 $3 \times 3$ & 6 & 96 & 2 & 1 \\
\hline $14^{2} \times 96$ & MBConv6 $5 \times 5$ & 6 & 192 & 4 & 1 \\
\hline $7^{2} \times 192$ & MBConv6 $3 \times 3$ & 6 & 320 & 1 & 1 \\
\hline $7^{2} \times 320$ & FC,Pooling & & & 10 & \\
\hline
\end{tabular}

TABLE I

WHERE T: EXPANSION FACTOR, C: NUMBER OF OUTPUT CHANNELS, N: NUMBER OF BLOCKS AND S: STRIDE

\section{A-MnasNet Architecture}

This section will explain the proposed architecture, AMnasNet, and the modifications made to the baseline architecture MnasNet.

\section{A. Convolution Layers}

MnasNet uses Depthwise Separable Convolutions. It has two layers of convolution. First, a $3 \times 3$ or $5 \times 5$ depthwise convolution layer. Second, a $1 \times 1$ pointwise convolution layer. The spatial dimensions along this convolution blocks remain constant. To increase the accuracy of the model, the convolutions must extract features in an efficient way. The channel expansion-contraction transformation throughout the architecture results in very wide feature maps in the middle of the building block. This increases the computational burden of such layers.

In order to reduce these computations, new convolutional layers were introduced in the model which are known as Harmonious Bottleneck Layers [2]. These layers focus on the spatial transformations along with channel transformations. They change the spatial feature scale along composite layers of the network. This strikes an improved balance between

\begin{tabular}{|c|c|c|c|c|c|}
\hline \multicolumn{6}{|c|}{ A-MnasNet Architecture } \\
\hline Layers & Convolutions & $\mathrm{t}$ & $\mathrm{c}$ & $\mathrm{n}$ & S \\
\hline $32^{2} \times 3$ & Conv $2 \mathrm{~d} 3 \times 3$ & - & 32 & 1 & 1 \\
\hline $112^{2} \times 32$ & SepConv $3 \times 3$ & 1 & 16 & 1 & 2 \\
\hline $112^{2} \times 16$ & MBConv3 $3 \times 3$ & 3 & 24 & 3 & 2 \\
\hline $112^{2} \times 4$ & Harmonious Bottleneck & 2 & 36 & 1 & 1 \\
\hline $56^{2} \times 36$ & MBConv3 $5 \times 5$ & 3 & 40 & 3 & 2 \\
\hline $112^{2} \times 40$ & Harmonious Bottleneck & 2 & 72 & 1 & 2 \\
\hline $28^{2} \times 72$ & MBConv6 $5 \times 5$ & 6 & 80 & 3 & 2 \\
\hline $112^{2} \times 80$ & Harmonious Bottleneck & 2 & 96 & 4 & 2 \\
\hline $14^{2} \times 96$ & MBConv6 $3 \times 3$ & 6 & 96 & 2 & 1 \\
\hline $14^{2} \times 96$ & MBConv6 $5 \times 5$ & 6 & 192 & 4 & 1 \\
\hline $7^{2} \times 192$ & MBConv6 $3 \times 3$ & 6 & 320 & 1 & 1 \\
\hline $7^{2} \times 320$ & FC,Pooling & & & 10 & \\
\hline
\end{tabular}

TABLE II

WHERE T: EXPANSION FACTOR, C: NUMBER OF OUTPUT CHANNELS, N: NUMBER OF BLOCKS AND S: STRIDE

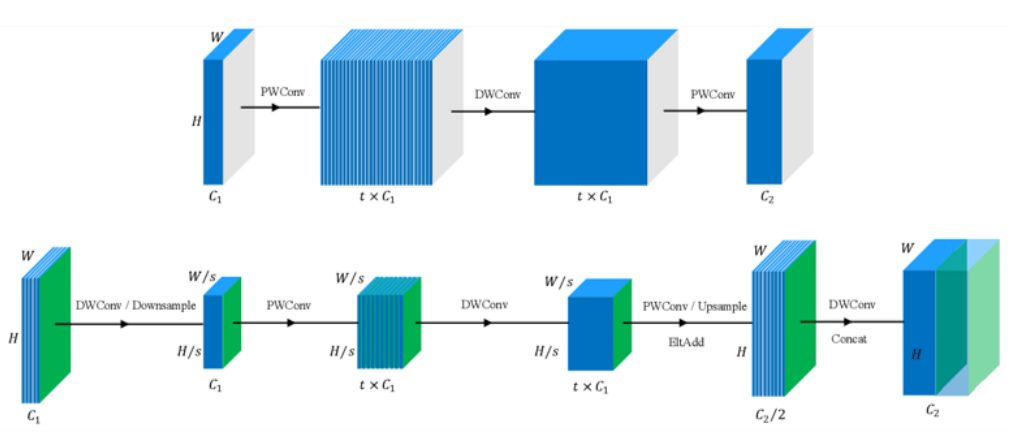

Fig. 2. Comparison of Depthwise Separable Convolution Layer and Harmonious Bottleneck Layer. [2]

the representation capability and efficiency by considering the spatial transformation.

As shown in Figure 2, there is contraction-expansion of spatial dimensions and expansion-contraction of channel dimensions in the harmonious bottleneck layer. First, the channel dimensions are kept constant and features are extracted from the spatial dimensions. Second, the spatial dimensions are kept constant and features are extracted from channel dimensions. This makes the convolution layer more efficient and increases the accuracy of the model.

Figure 2 shows the comparison of depthwise separable convolutional block and harmonious bottleneck layer. The spatial size of input/output feature maps is ( $\mathrm{H} \mathrm{x} \mathrm{W}), \mathrm{C} 1 / \mathrm{C} 2$ are input/output feature channels, $(\mathrm{K} \times \mathrm{K})$ is the kernel size and $\mathrm{s}$ denotes stride.

The total cost of depthwise separable convolution is

$$
(H \times W \times C 1 \times K \times K)+(H \times W \times C 1 \times C 2)
$$

The total cost of harmonious bottleneck layer is

$$
B / s^{2}+(H / s \times W / s \times C 1+H \times W \times C 2) \times K^{2}
$$

where, B is the computational cost of the blocks inserted between the spatial contraction and expansion operations. It is evident that by squeezing the channel expansion-contraction component and using a pair of spatial transformations yields a 
slimmed spatial size of wide feature maps in each stage which reduces the computational cost.

In this paper, three harmonious bottleneck layers were added to the MnasNet architecture to increase the accuracy. The model is described in table II. After adding these layers, the accuracy of the model increased by $12.17 \%$ whereas the model size decreased from 12.7 MB to 11.6 MB. The comparison is shown in Table III.

\section{B. Learning Rate Annealing or Scheduling}

While training a network, different learning rates are used to increase its accuracy. According to a pre-defined schedule, the learning rate is reduced while training the model. Some techniques like step decay, time decay, exponential decay and cosine annealing are very famous. Figure 3 illustrates step decay based learning rate performs better than other learning rate schedule methods. Therefore, this method is used for training A-MnasNet.

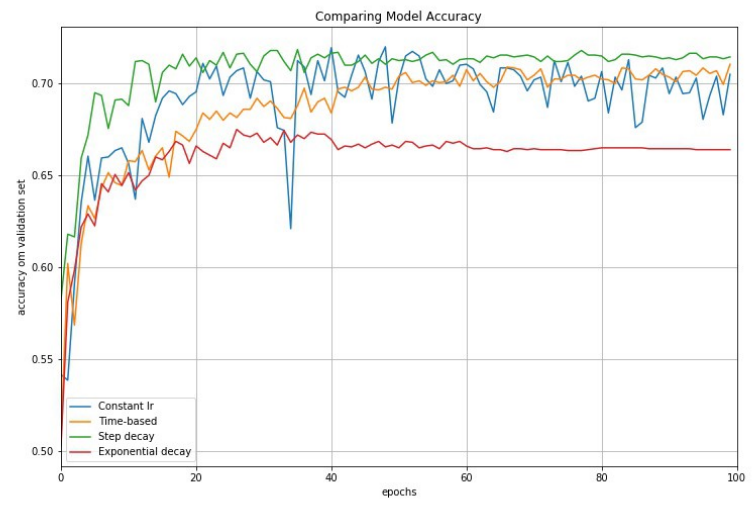

Fig. 3. Comparision of different LR scheduling methods. [33]

\section{Optimization}

Different optimization techniques can be used to optimize the performance of the network. Adaptive Learning Rate Method (Adadelta) [31], Adaptive Gradient Learning Algorithm (Adagrad) [31], Adaptive Moment Estimation technique (Adam) [31], Stochastic Gradient Descent method [31] and many more optimizers are widely used. RMSprop was used to optimize the MnasNet architecture. In this paper, SGD was implemented with varying values of learning rates and momentum $=0.9$ for better optimization.

\section{Data Augmentation}

AutoAugment [3] was used for data augmentation. AutoAugment learns the best augmentation policies for a given dataset with the help of Reinforcement Learning (RL). A policy consists of 5 sub-policies and each sub-policy applies 2 image operations in sequence. Each of those image operations has two parameters: The probability of applying it and the magnitude of the operation (e.g. rotate 20 degrees in $65 \%$ of cases). There is a controller that decides the best data augmentation policy at that instant and tests the generalization ability of that policy by running a child model experiment on a small subset of a particular dataset. After the child experiment is finished the controller is updated with the validation accuracy as the reward signal, using a policy gradient method called Proximal Policy Optimization algorithm (PPO). In this paper, AutoAugment is used on CIFAR-10 dataset. The accuracy of A-MnasNet was $92.97 \%$ but after using AutoAugment the accuracy increased to $96.89 \%$.

\section{HARDWARE AND SOFTWARE}

- Intel i9 9th generation processor with 32 GB RAM.

- Aorus Geforce RTX 2080Ti GPU

- Python version 3.6.7

- Pytorch version 1.0

- Spyder version 3.6

- Livelossplot (Loss and accuracy visualization).

\section{Results}

After making the aforementioned modifications to the baseline architecture, the model was trained with CIFAR-10 dataset on Aorus Geforce RTX 2080Ti GPU using PyTorch framework for 200 epochs. The data was divided into batch size of 128 for training set and batch size of 64 for validation set.

Figure 4 and figure 5 shows the plots of $\log \operatorname{loss}$ and accuracy of their respective models. The graphs were plotted using livelossplot visualization library.

The trade-off between model size and accuracy is shown in table III
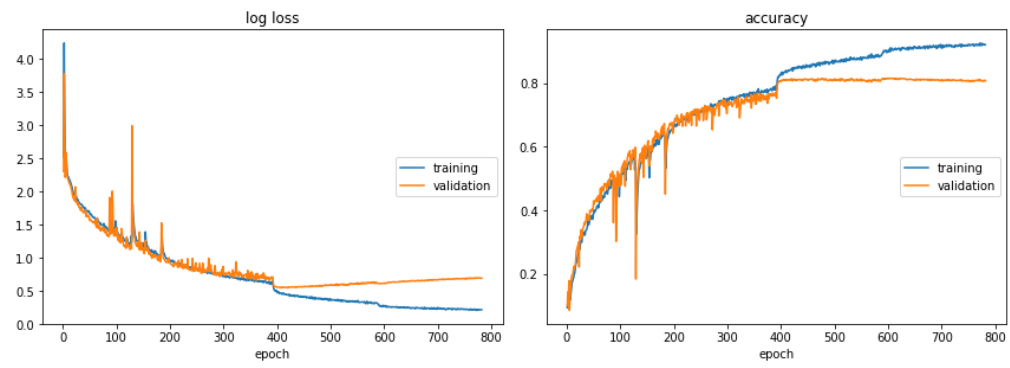

Fig. 4. MnasNet (Baseline Architecture)
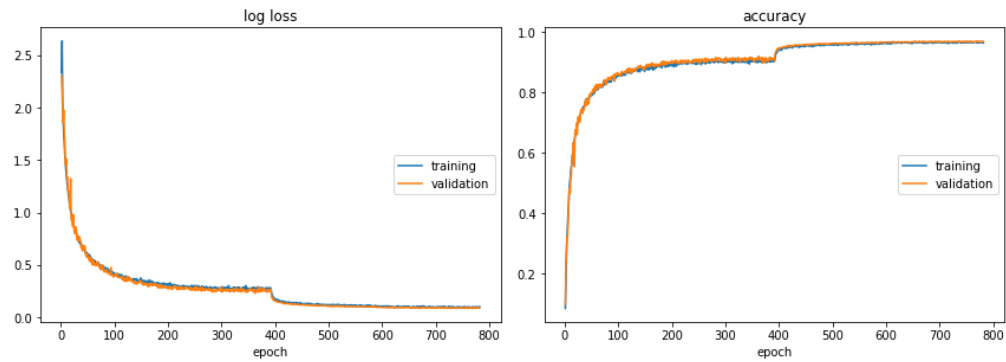

Fig. 5. A-MnasNet (Proposed Architecture)

Table IV shows the results obtained by scaling the model with different values of width multiplier. 


\begin{tabular}{|l|l|l|}
\hline \multicolumn{3}{|c|}{ Comparison of models } \\
\hline Architecture & Model Accuracy & Model size (in MB) \\
\hline MnasNet & $80.8 \%$ & 12.7 \\
\hline A-MnasNet & $96.89 \%$ & 11.6 \\
\hline
\end{tabular}

TABLE III

COMPARISON WITH BASELINE ARCHITECURE

\begin{tabular}{|l|l|l|}
\hline \multicolumn{3}{|c|}{ Scaling A-MnasNet with width multiplier } \\
\hline Width Multiplier & Model Accuracy & Model size (in MB) \\
\hline 1.4 & $97.16 \%$ & 22 \\
\hline 1.0 & $96.89 \%$ & 11.6 \\
\hline 0.75 & $96.64 \%$ & 6.8 \\
\hline 0.5 & $95.74 \%$ & 3.3 \\
\hline 0.35 & $93.36 \%$ & 1.8 \\
\hline
\end{tabular}

TABLE IV

VARYING MODEL SIZE BY WIDTH MULTIPLIER

\section{CONCLUSION}

In this paper, A-MnasNet, a new architecture is introduced by modifying MnasNet, its baseline architecture. The prime goal of this paper is to make the model more efficient in terms of accuracy. The accuracy of A-MnasNet is $96.89 \%$ with a size of $11.6 \mathrm{MB}$. It outperforms its baseline architecture MnasNet which has an accuracy of $80.8 \%$ and model size of 12.6 MB. Three new layers were added to the baseline architecture. These layers are called Harmonious Bottleneck layers. AutoAugment was used to further increase the accuracy of the model. This model can be implemented on mobile devices and other embedded systems. It can be used for applications like image classification, object detection and semantic segmentation.

\section{REFERENCES}

[1] Mingxing Tan, Bo Chen, et al. "MnasNet: Platform-Aware Neural Architecture Search for Mobile" arXiv:1807.11626v3 [cs.CV] 29 May 2019

[2] Duo Li, Aojun Zhou, Anbang Yao. "HBONet: Harmonious Bottleneck on Two Orthogonal Dimensions" arXiv:1908.03888v1 [cs.CV] 11 August 2019

[3] Ekin D. Cubuk, Barret Zoph, Dandelion Mane, Vijay Vasudevan, QuocV. Le Google Brain, "AutoAugment: Learning Augmentation Strategies from Data" arXiv:1805.09501v3 (2019)

[4] https://www.cs.toronto.edu/ kriz/cifar.html

[5] A. Krizhevsky, I. Sutskever, and G. E. Hinton, " Imagenet classification with deep convolutional neural networks" In Advances in neural information processing systems, pages 10971105, 2012.

[6] Howard, Andrew G., et al. "Mobilenets: Efficient convolutional neural networks for mobile vision applications." arXiv:1704.04861 (2017).

[7] Mark Sandler, Andrew Howard, Menglong Zhu, Andrey Zhmoginov, Liang-Chieh Chen. "MobileNetV2: Inverted Residuals and Linear Bottlenecks." arXiv:1801.04381v4 (2019)

[8] O. Russakovsky, J. Deng, H. Su, J. Krause, S. Satheesh, S. Ma, Z.Huang, A. Karpathy, A. Khosla, M. Bernstein, et al. "Imagenet largescale visual recognition challenge", International Journal of Computer Vision, 2015.

[9] J. Wu, C. Leng, Y. Wang, Q. Hu, and J. Cheng. "Quantized convolutionalneural networks for mobile devices". arXiv preprint arXiv: $1512.06473,2015$

[10] W. Chen, J. T. Wilson, S. Tyree, K. Q. Weinberger, and Y.Chen. "Compressing neural networks with the hashing trick". CoRR,abs/1504.04788, 2015

[11] S. Han, H. Mao, and W. J. Dally.’Deep compression: Compressing deep neural network with pruning, trained quantization and huffman coding”.CoRR, abs/1510.00149, 2, 2015.
[12] Karen Simonyan, Andrew Zisserman."VERY DEEP CONVOLUTIONAL NETWORKS FOR LARGE-SCALE IMAGE RECOGNITION" arXiv preprint arXiv:1409.1556v6 (2015)

[13] C. Szegedy, V. Vanhoucke, S. Ioffe, J. Shlens, and Z. Wojna. "Rethinking the inception architecture for computer vision." arXiv preprint arXiv:1512.00567, 2015.

[14] C. Szegedy, S. Ioffe, and V. Vanhoucke. Inception-v4, inception-resnet and the impact of residual connections on learning. arXiv preprintarXiv:1602.07261, 2016

[15] Sebastian Ruder. "An overview of gradient descent optimization algorithms." arXiv preprint arXiv:1609.04747, 2017

[16] F. N. Iandola, M. W. Moskewicz, K. Ashraf, S. Han, W. J. Dally, and K.Keutzer. Squeezenet: Alexnet-level accuracy with 50x fewer parameters and 1MB model size. arXiv preprint arXiv:1602.07360, 2016.

[17] Amir Gholami, Kiseok Kwon, Bichen Wu, Zizheng Tai, Xiangyu Yue, Peter Jin, Sicheng Zhao, Kurt Keutzer "SqueezeNext: Hardware-Aware Neural Network Design." arXiv preprint arXiv:1803.10615v2

[18] Kaiming He, Xiangyu Zhang, Shaoqing Ren, Jian Sun "Deep Residual Learning for Image Recognition." arXiv preprint arXiv: 1512.03385 (2015).

[19] Chen Wang, Yang Xi. Convolutional Neural Network for Image Classification.

[20] MD. ZAKIR HOSSAIN, FERDOUS SOHEL, MOHD FAIRUZ SHIRATUDDIN, HAMID LAGA (2018). "A Comprehensive Survey of Deep Learning for Image Captioning." arXiv preprint arXiv: 1810.04020 .

[21] Zhong-Qiu Zhao, Shou-tao Xu, and Xindong Wu. "Object Detection with Deep Learning: A Review." arXiv preprint arXiv:1807.05511 (2019).

[22] Abien Fred M. Agarap. "Deep Learning using Rectified Linear Units (ReLU)." arXiv preprint arXiv:1803.08375v2[cs.NE], 2019

[23] Xiangyu Zhang, Xinyu Zhou, Mengxiao LinJian, Sun. "ShuffleNet: An Extremely Efficient Convolutional Neural Network for Mobile." arXiv preprint arXiv:1707.01083v2, 2017

[24] Francois Chollet (2017). "Xception: Deep Learning with Depthwise Separable Convolutions. arXiv preprint arXiv: 1610.02357

[25] Zhun Zhong, Liang Zheng, Guoliang Kang, Shaozi Li, Yi Yang (2017). "Random Erasing Data Augmentation." arXiv preprint arXiv:1708.04896

[26] Barret Zoph, Ekin D. Cubuk, Golnaz Ghiasi, Tsung-Yi Lin, Jonathon Shlens, Quoc V (2019). Le. "Learning Data Augmentation" arXiv preprint arXiv: 1906.11172

[27] Sergey Ioffe and Christian Szegedy. Batch Normalization: Accelerating Deep Network Training by Reducing Internal Covariate Shift." arXiv preprint arXiv: $1502.03167,2015$

[28] Timothy Dozat (2016). "INCORPORATING NESTEROV MOMENTUMINTO ADAM." Workshop track-ICLR 2016.

[29] Diederik Kingma and Jimmy Ba. "Adam: A method for stochastic optimization." arXiv preprint arXiv:1412.6980, 2014

[30] Barret Zoph, Vijay Vasudevan, Jonathon Shlens and Quoc V. Le, "Learning Transferable Architectures for Scalable Image Recognition", arXiv:1707.07012v4 [cs.CV] 11 Apr 2018.

[31] Sebastian Ruder, "An overview of gradient descent optimization algorithms*",arXiv:1609.04747v2 [cs.LG] 15 Jun 2017

[32] Nitish Srivastava, Geoffrey Hinton, Alex Krizhevsky, Ilya Sutskever, Ruslan Salakhutdinov, "Dropout: A Simple Way to Prevent Neural Networks from Overfitting", Journal of Machine Learning Research 15 (2014) 1929-1958, Submitted 11/13; Published 6/14

[33] https://towardsdatascience.com/learning-rate-schedules-and-adaptivelearning-rate-methods-for-deep-learning-2c8f433990d1 